\title{
The Three-Dimensional Structure of EUV Accretion Regions of AM Her Stars: Analysis of $E U V E$ Light Curves
}

\author{
MARTIN M. SIRK ${ }^{1}$ AND STEVE B. HOWELL ${ }^{2}$ \\ ${ }^{1}$ Center for EUV Astrophysics, 2150 Kittredge St., \\ University of California, Berkeley, CA 94720-5030, USA \\ ${ }^{2}$ Planetary Sciences Institute, Astrophysics Group, \\ 620 North Sixth Ave., Tucson, AZ 85705, USA
}

\begin{abstract}
We present EUV light curves for a number of AM Her systems observed either as guest observer targets or with the EUVE Right Angle Program. We have formed light curves for eight AM Her stars and show in our presentation the similarities and differences present. We draw some conclusions by grouping the systems by inclination, magnetic field strength, and accretion region geometry. In order to understand the physical structures responsible for the EUV emissions, we have developed a software model to generate synthetic light curves. We find that the EUV accretion regions in the systems UZ For, VV Pup, and AM Her cannot be fit with a flat spot confined to the white dwarf surface, regardless of its shape or brightness profile. Rather, a small, symmetric, raised spot is the only shape consistent with the data. The light curves for systems EF Eri, RE1149+28, AN UMa, and V834 Cen show evidence for additional structure that precedes the primary accretion region in phase. Our model indicates that a large portion of the light curve in each system is seen in absorption. Finally, in three systems, we detect a very gradual rise and fall in the EUV flux ( $<5 \%$ of the peak flux) at phases when the spot is completely obscured behind the white dwarf (WD) limb. We attribute this detection to emission from the accretion column that decreases exponentially with distance from the WD and detect emission at heights up to $15 \%$ of the WD radius.
\end{abstract}

\section{Introduction and Data}

AM Her stars are a class of interacting binaries consisting of a highly magnetic (order $10-60 \mathrm{MG}$ ) white dwarf (WD) primary and a red dwarf secondary that fills its Roche Lobe. Stellar material flows through the inner Lagrangian point and falls toward the primary forming an accretion stream. In the absence of a magnetic field, an accretion disk normally forms. However, in AM Her systems, the strong magnetic field captures the ionized material and channels it directly toward one or both of the magnetic poles of the WD primary, forming a hot accretion spot. The interaction of the accretion stream with the magnetic field circularizes the orbits and synchronizes the WD rotation period with the binary orbital period. When viewed from the rotating frame, the primary, secondary, and magnetic field all appear static. The only motion is that of the in-falling material, its free-fall time being about one fourth the orbital period.

Conversion of the kinetic energy of the stream manifests itself in many forms. Electrons in the stream near the spot spiral around the field lines and emit highly polarized cyclotron radiation at optical and near infrared wavelengths. X-rays arise from the post shock region where the supersonic stream meets the WD photosphere. Extreme ultraviolet (EUV) radiation is detected on, or very close to, the heated WD surface; the consequence of reprocessed X-rays and the direct mechanical heating of the WD photosphere by the impacting material. See Liebert \& Stockman (1985) and Cropper (1990) for reviews on AM Her systems.

Observations in the EUV are particularly well suited to probing the accretion region 

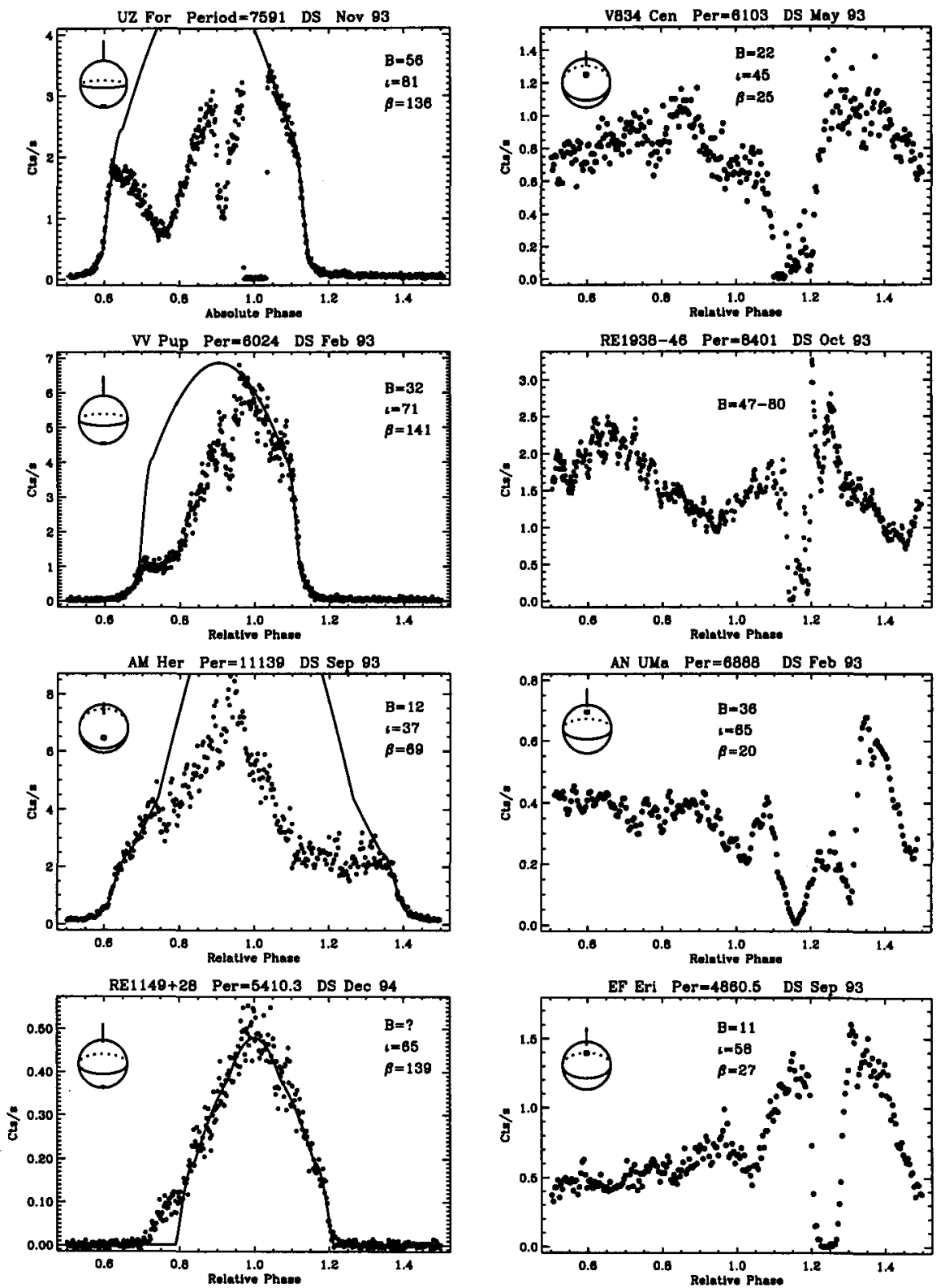

FIgure 1. Phase folded light curves of eight AM Her systems. Geometric models are fit to the stars in the left column with the derived inclinations $\iota$ and spot colatitudes $\beta$ indicated. Models have not yet been fit to the stars in the right column since their light curves show additional structure. Here, $\iota$ and $\beta$ are from Cropper (1990), as are all the periods (seconds) and magnetic fields $B$ (MG). 

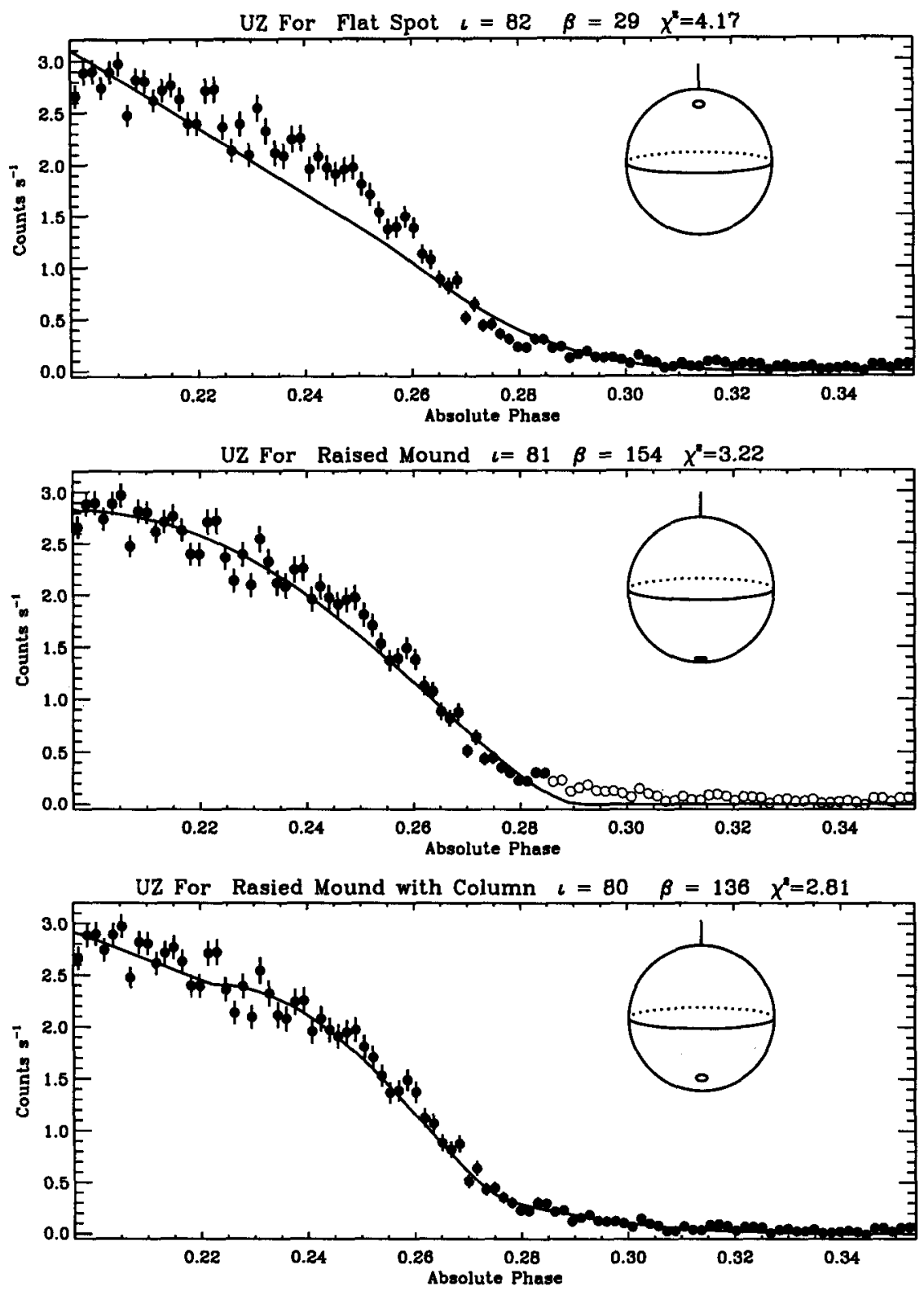

FIGURE 2. Enlargement of the EUV fall phase of UZ For comparing three different models. Only the raised-mound model with luminous accretion column shows no systematic residuals (bottom panel). 


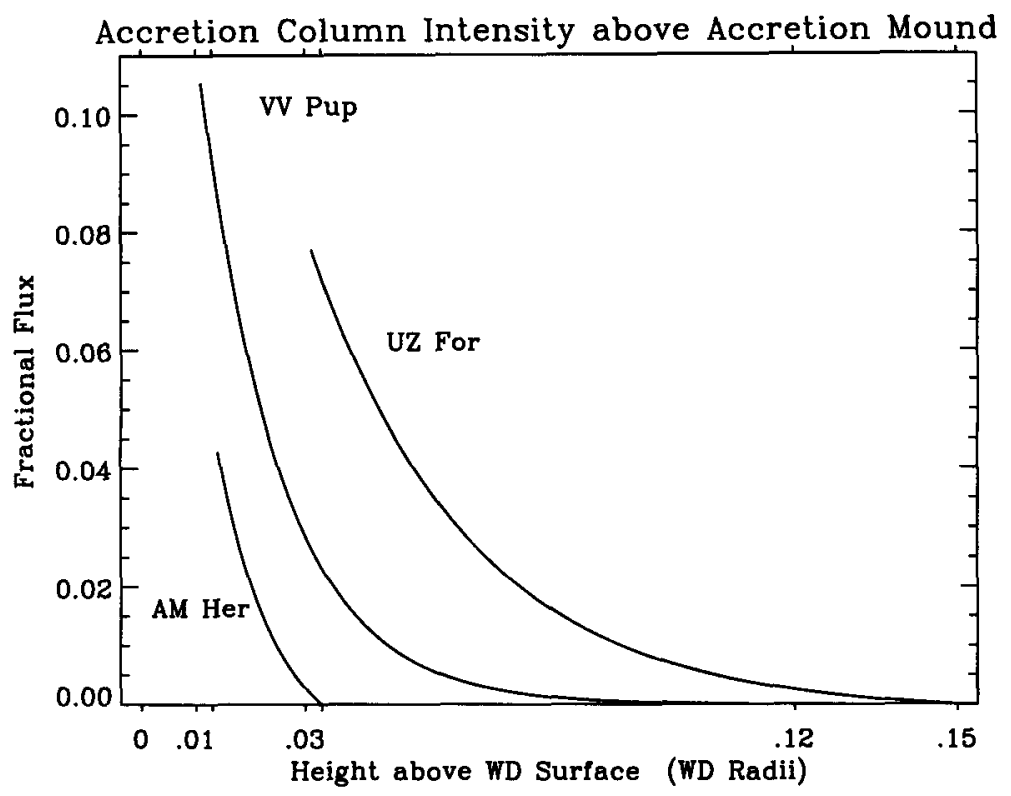

FIGURE 3. Accretion column intensity as a function of distance from the WD surface for UZ For, VV Pup, and AM Her. The column intensities are normalized to the peak intensity for each star.

since the source of radiation is often confined to a very small region on, or near, the WD surface. Furthermore, the systems are intrinsically bright around $100 \AA$.

Analysis of Extreme Ultraviolet Explorer (EUVE ; Bowyer \& Malina 1991) photometry of UZ For (Warren, Sirk, \& Vallerga 1995) showed a very small, symmetric EUV accretion spot that is raised a few percent above the WD surface. Vennes et al. (1995) determined a similar height for the accretion spot of VV Pup based on model atmosphere calculations. Vertical extension of the accretion region profoundly affects the EUV light curves, both by lengthening the duration of the bright phase (when the spot is not behind the WD limb), and steepening the rise and fall phases (when the spot rotates into and out of view from behind the WD limb). Understanding the physical processes of AM Her systems requires accurate knowledge of the system geometry, namely the orbital inclination $(\iota)$ and the colatitude $(\beta)$ of the accretion spot. These parameters are often determined from optical light curves. Assuming a flat accretion spot may result in incorrect values of $\iota$ and $\beta$. Thus, we decided to model the EUV accretion regions, allowing the inclination, spot colatitude, spot size, and spot height to all be free parameters.

Utilizing our own EUVE guest observer data (Howell et al. 1995), data from the EUVE public archive, and data from the EUVE Right Angle Program (McDonald et al. 1994) we construct high time-resolution, phased-folded light curves for eight AM Her systems obtained by the Deep Survey and scanner telescopes. All data are in the Lexan/boron passband ( $\geq 10 \%$ transmission from 67 to $178 \AA$, peak at $91 \AA$; Malina et al. 1994). We present the light curves as Figure 1. 


\section{TABLE 1. System Parameters}

$\begin{array}{lcccccccc}\text { System } & \begin{array}{c}\text { Period } \\ (\min )\end{array} & \begin{array}{c}\iota \\ \left(^{\circ}\right)\end{array} & \begin{array}{c}\beta \\ \left({ }^{\circ}\right)\end{array} & \begin{array}{c}r \\ (\mathrm{WD})\end{array} & \begin{array}{c}h \\ (\mathrm{WD})\end{array} & \begin{array}{c}h_{\text {col }} \\ (\mathrm{WD})\end{array} & \begin{array}{c}\psi \\ \left(^{\circ}\right)\end{array} & \begin{array}{c}B \\ (\mathrm{MG})\end{array} \\ \text { UZ FordS } & 126.5 & \mathbf{8 0 . 5} & \mathbf{1 3 6 . 1} & .059 & .090 & .15 & 49 & 56 \\ \text { UZ Forscan } & 126.5 & \mathbf{8 0 . 5} & \mathbf{1 1 6 . 2} & .078 & .019 & .14 & 55 & 56 \\ \text { VV Pup } & 100.4 & \mathbf{7 0 . 6} & \mathbf{1 1 1 . 2} & .022 & .010 & .12 & 49 & 32 \\ \text { AM Her } & 185.6 & \mathbf{3 6 . 9} & \mathbf{6 8 . 6} & .045 & .019 & .033 & 31 & 12 \\ \text { RE1149+28 } & \mathbf{9 0 . 1 7} & \mathbf{6 5} & \mathbf{1 9 9} & .069 & .016 & - & - & - \\ \text { EF Eri } & 81.01 & \mathbf{7 4} & \mathbf{7 4} & .061 & .015 & - & - & 11\end{array}$

NotEs: The system inclination is $\iota$. The angle between the rotational pole and the EUV accretion spot is $\beta$. The radius and the height of the accretion spot are $r$ and $h$, respectively, in units of WD radii. The maximum height of the accretion column above the WD surface that shows significant flux is $h_{\text {col }}$ in units of WD radii. The longitude of the accretion spot is $\psi$, and the field strength of the primary magnetic pole is $B$. Italic entries for $\iota, \beta, r, h$, and $h_{\text {col }}$ are the parameter solutions derived from the model fits (light face entries indicate that that particular parameter was fixed, not free). Other italic entries are values determined from $E U V E$ data. The remaining light face entries are from Cropper's (1990) review article, The Polars.

\section{EUV Accretion Spot Models}

Three symmetric spot models were attempted: a flat circular spot, a cone with circular base, and a raised mound (sector of rotation). The synthetic light curves from a flat spot closely resemble a cosine curve, and thus, completely fail to account for the steep rise and fall phases of the EUV light curves. The conical spot proved only slightly better. The raised mound, however, fit the data very nicely. We started with UZ For since its inclination and spot colatitude were accurately determined from optical eclipse photometry by Bailey \& Cropper (1991), and the EUV spot size from eclipse timings (Warren et al. 1995). To test the applicability of the model, the inclination and the spot colatitude were allowed to be free parameters, even though they were already known. Fitting UZ For, we find a spot radius and spot height of .06 and $.03 \mathrm{WD}$ radii, respectively, and an inclination and colatitude of 80 and 136 degrees, in excellent agreement with Bailey \& Cropper (1991). The same model is applied to VV Pup, AM Her, and RE1149+28. The derived parameters are listed in Table 1 in italic bold face.

Our raised-mound model fits the steep rise and fall regions of the light curves quite well. The central parts of each light curve are subject to eclipses by the secondary star, eclipses by the accretion stream, and absorption by material very close to the accretion spot, and thus, are excluded from the model fits. A careful study of the residuals of the four fit stars showed a systematic and symmetric underestimate of the flux at the earliest and latest segments of the rise and fall phases, respectively. Rather than invoking a complicated horizontal brightness profile of the accretion spot that symmetrically leads and follows the spot center, we attribute the residual flux to EUV radiation emanating from the accretion column immediately above the accretion spot proper (the residual flux is $1 \%-2 \%$ of the peak flux). Only two additional parameters (a brightness scale factor, and an exponential decay constant) are required to adequately model the light curves. The maximum height of the EUV luminous accretion column may be measured directly from the light curves once $\iota$ and $\beta$ are known. Figure 2 compares the fits of a flat spot, a raised mound, and a raised mound with luminous accretion column to the fall phase of UZ For (top, middle, and bottom panels, respectively). The flat spot fit shows large residuals and places the accretion spot $130^{\circ}$ away (in the opposite hemisphere) 
from its known location. The raised-mound fit does well in the steep fall phase but systematically misses the last portion of the fall phase (plotted as open circles in the middle plot). The final panel includes the accretion column as a source of EUV flux that decays exponentially with height above the WD surface. All points in the rise and fall phase are fit by this model.

In Figure 3 we plot the derived accretion column intensity as a function of distance above the WD for three systems, normalized to the peak intensity measured for each system. UZ For shows column flux at a maximum height of .15 the WD radius.

\section{Conclusions}

AM Her stars that show symmetric EUV light curves in their rise and fall phases are consistent with small, symmetric accretion regions that are typically $2 \%-6 \%$ of the WD radius in width and $1 \%-3 \%$ of the WD radius in height. These systems do not require additional structures, such as second emitting poles, auroral arcs, or complicated horizontal brightness profiles. Flux detected at the very early rise and very late fall phases may be explained by a luminous accretion column that decreases exponentially in intensity with distance from the WD. The system inclination and accretion spot colatitude may be directly determined from model fits of the light curves. Conversely, if these last two parameters are already known from other sources, the models are constrained, improving the estimates of the three dimensional structure of the EUV accretion region.

The sections of the light curves where the geometric models fail (in phases during which the spot is seen mainly face on) are indicative of additional structure. Analysis of phased resolved spectra will address the question of whether the "missing" flux is the result of absorption, or genuine structure in the accretion region when viewed face on. We are refining our techniques in effort to model the systems of the right side of Figure 1 , and are investigating the time variability of the EUV light curves.

We thank the CEA Guest Observer Center support personnel. This work is funded in part by NASA contract NAS5-30180. SBH acknowledges support from NASA grant NAG5-2902.

\section{REFERENCES}

BAIley, J. \& Cropper, M. S. 1991, MNRAS, 253, 27

BowYer, S. \& Malina, R. F. 1991, in Extreme Ultraviolet Astronomy, ed. R. F. Malina \& S. Bowyer, New York: Pergamon Press, 397

Cropper, M. S. 1990, Space Sci. Rev., 54, 195

Howell, S. B., Sirk, M. M., Malina, R. F., Mittaz, J. P. D., \& Mason, K. O. 1995, ApJ, 439, 991

LieberT, J. \& Stockman, H. S. 1985, in Cataclysmic Variables and Low-Mass X-ray Binaries, ed. D. Q. Lamb \& J. Patterson, Dordrecht: Reidel, 151

Malina, R. F., ET AL. 1994, AJ, 107, 751

MCDonald, K., ET AL. 1995, AJ, 108, 5

Vennes, S., SzKody, P., Sion, E. M., \& Long, K. S. 1995, ApJ, 445, 921

Warren, J. K., Sirk, M. M., \& Vallerga, J. V. 1995, ApJ, 445, 909 